\title{
Degradation of thin tungsten filaments at high temperature in HWCVD
}

\author{
$\underline{\text { P.A. Frigeri }}{ }^{1}$, O. Nos ${ }^{1}$, J. Bertomeu $^{1}$ \\ ${ }^{1}$ Dept. de Física Aplicada i Òptica, Universitat de Barcelona \\ Barcelona-08028, Spain, Ph: +34934039223 , fax: +34934039219 \\ Email of corresponding author: pfrigeri@ub.edu
}

\begin{abstract}
The degradation of the filaments is usually studied by checking the silicidation or carbonization status of the refractory metal used as catalysts, and their effects on the structural stability of the filaments. In this paper, it will be shown that the catalytic stability of a filament heated at high temperature is much shorter than its structural lifetime. The electrical resistance of a thin tungsten filament and the deposition rate of the deposited thin film have been monitored during the filament aging. It has been found that the deposition rate drops drastically once the quantity of dissolved silicon in the tungsten reaches the solubility limit and the silicides start precipitating. This manuscript concludes that the catalytic stability is only guaranteed for a short time and that for sufficiently thick filaments it does not depend on the filament radius.
\end{abstract}

\section{Keywords}

Hot wire chemical vapor deposition; catalytic chemical vapor deposition; silicon; filament degradation; filament lifetime; catalytic activity. 


\section{Introduction}

The problem of filament degradation, also known as filament ageing, in Hot Wire CVD (HWCVD) processes constitutes the major limiting factor for the industrial implementation of this technique. In the particular case of silicon deposition, using for instance silane $\left(\mathrm{SiH}_{4}\right)$ as precursor gas, the degradation of the catalytic filaments (usually made of tungsten or tantalum) is due to the formation of silicides. Several studies dealing with the silicidation process of filaments during amorphous $(\mathrm{a}-\mathrm{Si}: \mathrm{H})$ or microcrystalline silicon $(\mu \mathrm{c}-\mathrm{Si}: \mathrm{H})$ deposition with HWCVD have been published in the last decade for either tantalum [1-5] or tungsten [2, 5-9] filaments. Alternatively, the protection of the filaments against silicidation has been dealt in some previous works [2, $4,10-12]$. In these prior manuscripts, structural filament lifetimes, defined as the usage time until the filament breakage occurs, up to a maximum of few hundred hours have been reported. However, in these latter manuscripts little or no attention has been paid to the catalytic performance of the filaments during its usage time [7]. In any precedent work, the degradation of the catalytic activity has not been correlated with the state of the filament. In this manuscript, the term catalytic activity is interpreted as the reaction rate $K$ of the decomposition of silane in its primary radicals per unit length of filament. The filament temperature $\left(T_{f}\right)$ dependence of the reaction rate is given by

$$
K=K_{0} \cdot \exp \left[-\frac{E_{a}}{R \cdot T_{f}}\right]
$$

Where $R$ is the gas constant, $E_{a}$ is the activation energy (expressed in $\mathrm{J} / \mathrm{mol}$ ), and, $K_{0}$ is a function of the filament radius, and of the partial pressures of the different radical and gases species present in the chamber. 
The scope of this work is to clarify the relationship between the beginning of the catalytic performance decay of a filament heated at high temperature and the chemical composition of the catalyst surface. This objective was achieved by applying simultaneously two different procedures to check, from one side, the stability of the catalyst, and from the other side, the chemical composition of the filament surface. The stability of the catalyst was simply checked by comparing the thickness of films deposited during regular subsequent intervals. To be able to deduce the chemical composition of the filament surface during these deposition intervals, we needed to modelize the electric resistance change of a filament during different stages of its degradation. This allowed us to interpret an abrupt change observed during the monitoring of the electric resistance.

\section{Electric model for an ageing filament}

During the degradation process of a filament, silicon atoms diffuse concentrically towards the filament core. A used tungsten filament goes through an initial stage of Si dissolution forming a solid solution, which is followed by the precipitation of $\mathrm{W}_{5} \mathrm{Si}_{3}$

once the concentration of $\mathrm{Si}$ atoms reaches its solubility limit [6]. At higher $\mathrm{Si}$ concentrations, the precipitation of $\mathrm{WSi}_{2}$ may take place. The initial stage of $\mathrm{Si}$ dissolution is particularly important for filaments heated at temperatures higher than $180{ }^{\circ} \mathrm{C}$, when the solubility limit becomes appreciable. On the other hand, for filament temperatures lower than $1750{ }^{\circ} \mathrm{C}$ [8], the first two stages follow one another so quickly that the formed outer corona is practically exclusively composed by $\mathrm{WSi}_{2}$. This is the case of the cold portions of the filament located close to the electrical contacts, which 
are rapidly converted in $\mathrm{WSi}_{2}$. We have observed in our experiments that these $\mathrm{WSi}_{2}$ portions start expanding, after a first transient period, towards the middle of the filament with an almost constant propagation velocity (see Fig. 1).

Fig. 2 schematically shows the degradation process of a tungsten filament at high temperature $\left(>1800{ }^{\circ} \mathrm{C}\right)$, showing the $\mathrm{Si}$ atoms dissolution stage and the subsequent formation of a $\mathrm{W}_{5} \mathrm{Si}_{3}$ outer corona once the solubility limit has been reached. The different regions depicted in Fig. 2, correspond to the $\mathrm{WSi}_{2}$ portions expanding from the cold ends (a), a transition zone occupied by the $\mathrm{WSi}_{2}$ front (b) and the central part of the filament (c).

Taking all these into account, the variation of the electric resistance of the filament, after an initial lapse of time, $t_{i}$, necessary for the complete conversion of the cold ends into $\mathrm{WSi}_{2}$, can be written as

$$
R_{f i l} \approx 2 C+2 R^{\prime}{ }_{a} v\left(t-t_{i}\right)+R_{c}^{\prime}(t) \cdot\left(L_{f i l}-2 v\left(t-t_{i}\right)\right)
$$

where $R_{a}^{\prime}=R_{W S i_{2}}^{\prime}$ is the electric resistance per unit length of a filament portion which has been completely transformed into $\mathrm{WSi}_{2}$ and $\mathrm{R}_{\mathrm{c}}{ }_{\mathrm{c}}(\mathrm{t})$ corresponds to the resistance per unit length of the central part of the filament, $\mathrm{v}$ is the propagation velocity of the $\mathrm{WSi}_{2}$ fronts, $\mathrm{L}_{\mathrm{fil}}$ is the length of the central part of the filament and the term $C$ contains the resistance contributions, of the boundary region close to the electrical contact and of the $\mathrm{WSi}_{2}$ front. Here, we have used the term "central part of the filament" to denote that portion of the filament, not transformed into $\mathrm{WSi}_{2}$, for which all dissipated power is irradiated and no conduction losses are relevant to determine its temperature $T_{f}$. We will 
expose the case of a $0.125 \mathrm{~mm}$ diameter filament heated up to $1900{ }^{\circ} \mathrm{C}$ using a fixed current $I=1.63$ A.

Before the Si concentration reaches its solubility limit, which is of few percent at $1900{ }^{\circ} \mathrm{C}$, the central part of the filament can be considered exclusively made of tungsten. One finds that $R_{c}^{\prime}(t)$ is simply given by

$$
R_{c}^{\prime}(t)=R_{W}^{\prime}\left(1900^{\circ} \mathrm{C}\right)
$$

where $\mathrm{R}_{\mathrm{W}}\left(1900{ }^{\circ} \mathrm{C}\right)$ is the electric resistance per unit length of a tungsten filament at $1900{ }^{\circ} \mathrm{C}$. The temperature of the $\mathrm{WSi}_{2}$ portions will be about $350{ }^{\circ} \mathrm{C}$ lower due to the higher emissivity and the increase of the local radius, i.e. $R_{a}^{\prime}=R_{W S i_{2}}^{\prime}\left(1550{ }^{\circ} \mathrm{C}\right)$. This stage is characterized by a linear increasing of the filament resistance,

$$
\frac{d R_{f i l}}{d t} \approx 2 v\left(R^{\prime}{ }_{W S i_{2}}\left(1550^{\circ} \mathrm{C}\right)-R_{W}^{\prime}\left(1900{ }^{\circ} \mathrm{C}\right)\right)
$$

given by the advance of the two $\mathrm{WSi}_{2}$ fronts towards the middle of the filament. According to our calculations, $\mathrm{R}_{\mathrm{W}}^{\prime}\left(190{ }^{\circ} \mathrm{O}\right)=52 \pm 3 \Omega / \mathrm{m}$ and ${R^{\prime}}_{W S i_{2}}\left(1550{ }^{\circ} \mathrm{C}\right)=57 \pm 3$ $\Omega / \mathrm{m}$. These last have been evaluated by taking into account the temperature dependence of the resistivity and the emissivity of $\mathrm{W}$ and $\mathrm{WSi}_{2}[8,13-16]$, and also, the increasing of the filament radius in the $\mathrm{WSi}_{2}$ portion.

Once the solubility limits is reached at $t=t_{0}$, the silicide phase $\mathrm{W}_{5} \mathrm{Si}_{3}$ begins to precipitate at the surface of the filament. The first consequence of the precipitation is the change in the emissivity, passing from $e_{\mathrm{W}}$ to $e_{\mathrm{W} 5 \mathrm{Si} 3}$, what causes a temperature drop 
of about $150{ }^{\circ} \mathrm{C}$, estimated using the Stefan-Boltzmann law. Then, the silicide progressively consumes the central part of the tungsten filament, transforming it into $\mathrm{W}_{5} \mathrm{Si}_{3}$. A first order approximation of $\mathrm{R}_{\mathrm{c}}{ }_{\mathrm{C}}(\mathrm{t})$ is given by

$$
R_{c}^{\prime}(t) \approx R_{W}^{\prime}(1750 \stackrel{\circ}{\circ})+\left(\frac{R^{\prime}{ }_{W_{5}} S i_{3}\left(1750^{\circ} \mathrm{O}\right)-R^{\prime}{ }_{W}(1750 \stackrel{\circ}{\circ})}{t_{c}}\right) \cdot\left(t-t_{0}\right)
$$

for $t_{0}<t<t_{0}+t_{c}$, where $\mathrm{R}_{\mathrm{W}_{5} \mathrm{Si}_{3}}$ corresponds to the electric resistances per unit length of a filament portion exclusively composed by $\mathrm{W}_{5} \mathrm{Si}_{3}$ and $\mathrm{t}_{\mathrm{c}}$ is the amount of time needed by the $\mathrm{W}_{5} \mathrm{Si}_{3}$ outer layer to reach the filament core. During this phase, and considering only the linear term of $R_{f i l}(t)$, it can be seen from Eqs. 2, and 5, that $\mathrm{R}_{\mathrm{fil}}$ increases as

$$
\begin{aligned}
\frac{d R_{f i l}}{d t} & \approx 2 v\left(R^{\prime}{ }_{W i_{2}}\left(1550{ }^{\circ} \mathrm{C}\right)-R^{\prime}{ }_{W}\left(1750{ }^{\circ} \mathrm{C}\right)\right) \\
& +\left(\frac{R_{{ }_{5} S i_{3}}^{\prime}\left(1750^{\circ} \mathrm{C}\right)-R^{\prime}{ }_{W}\left(1750{ }^{\circ} \mathrm{C}\right)}{t_{c}}\right) \cdot\left(L_{f i l}-2 v t_{0}\right)
\end{aligned}
$$

for $t_{0}<t<t_{0}+t_{c}$. In this case, we have estimated $R^{\prime}{ }_{W}\left(1750{ }^{\circ} \mathrm{C}\right)=48 \pm 3 \Omega / \mathrm{m}$, and $R_{W_{5} S_{3}}^{\prime}\left(1750^{\circ} \mathrm{C}\right)=64 \pm 3 \Omega / \mathrm{m}$. The first term of the equation (6) is of the same order than that given by (4), since no change in $v$ was observed. But the second term can be considerable larger than the first one if the tested filament is sufficiently large and thin, because $t_{c}$ has a quadratic dependence with the filament radius [8]. This is the motivation of using thin filaments of only $0.125 \mathrm{~mm}$ diameter: To observe a clear change in the slope of the electrical resistance variation with time once the $\mathrm{W}_{5} \mathrm{Si}_{3}$ is precipitating. 


\section{Experimental details}

The filament deterioration study was carried out in a single chamber able to be supplied with silane and hydrogen gases, and equipped with a standard high-vacuum pumping system. The tests were performed mainly in a pure silane atmosphere at a partial pressure of $p_{\mathrm{SiH}_{4}}=2 \mathrm{~Pa}$, which is a typical pressure for the deposition of a-Si:H material.

A specific invented filament holder was used to connect the thin tungsten filament of $0.125 \mathrm{~mm}$ diameter and about $30 \mathrm{~cm}$ length to a direct electrical current source delivering 1.63 A to heat the filament up to about $1900{ }^{\circ} \mathrm{C}$. This initial filament temperature was estimated indirectly solving equation (7) for the given filament current and using tabulated resistivity and emissivity values for tungsten $[13,14]$. The change of the filament electrical potential was monitored during all the duration of the experiment, and the total resistance of the filament $R_{\text {fil }}(t)$ was determined. The filament was vertically oriented and the holder took advantage of the gravity force to keep straight the filament despite its thermal expansion.

It is important to notice that the filament was heated using a constant current I, and not using a constant voltage. This allows avoiding the temperature of the central part of the filament being influenced by the deterioration of the cold portions located close to the electrical contacts. In fact, at the central part of the filament, all dissipated power is irradiated and the filament temperature $T_{f}$, is given by Stefan-Boltzmann law

$$
\frac{\rho\left(T_{f}\right) \cdot I^{2}}{\pi \cdot r^{2}}=e \cdot \sigma \cdot \pi \cdot r \cdot T_{f}^{4}
$$


Here, the tungsten resistivity, $\rho$, the emissivity, $e$, and the radius of the filament, $r$, are local quantities, and $\sigma$ is just the Stefan-Boltzmann constant. But if the filament was heated using a constant voltage, the filament current $I$, would depend on the total resistance of the filament $R_{f i l}$, and the temperature of the central part of the filament $T_{f}$, would be influenced by the degradation at the cold ends.

The experimental set up was constructed to study only the catalytic variation of the central part of the filament. To do that, a metallic mask with an open window of about 3 $\mathrm{cm} \times 2 \mathrm{~cm}$, and surrounded with a special frame, was placed in front of the central part of the filament at a distance of about $1.5 \mathrm{~cm}$ to delimit the deposition area and to reduce the portion of filament contributing to the deposition. A glass substrate placed just behind the mask and mounted over a revolving support was used to feed, at regular intervals, the deposition area with a clean substrate portion. The substrate was kept at a constant temperature by means of an infrared heater. After the experiment, the thickness of the films deposited during each time interval $(15 \mathrm{~min}$ or $25 \mathrm{~min}$, depending on the performed silicidation test) was measured with an interferometric optical microscope (Sensofar, PL $\mu$ 2300).

\section{Result and Discussion}

Three filaments were tested using the procedure described in the previous section in a pure silane atmosphere. The structural lifetime was different for the three filaments. The propagation velocity of the $\mathrm{WSi}_{2}$ portions expanding from the cold ends was measured by means of direct observation giving a very similar value $v=(4.0 \pm 0.3)$. $10^{-4} \mathrm{~m} / \mathrm{min}$ for all filaments. The filaments electrical resistance $\mathrm{R}_{\mathrm{fil}}(\mathrm{t})$ as a function of 
time and the values of the normalized film thickness $d$, deposited in the corresponding time intervals, are shown in Fig. 3, and Fig. 4, respectively.

In Fig. 3 one can clearly recognize the electrical resistance signatures of the different stages characterizing the aging of the filament as described in section 2. After an initial transient period, of about $t_{i} \approx 20 \mathrm{~min}$, allR $\mathrm{fil}_{\mathrm{fil}}(\mathrm{t})$ curves enter alinear regime characterized by a slope of around $0.01 \Omega / \mathrm{min}$. This result is very close to the maximum value expected for the stage where the silicon is dissolved in the filament by forming a solid solution. In fact using Eq. 3, the estimated values for $R^{\prime}{ }_{W}, R^{\prime}{ }_{W S i_{2}}$ (introduced in section 2 ) and the velocity $v$, measured experimentally, the maximum slope expected for this stage is $0.009 \Omega / \mathrm{min}$.

At $t_{0} \approx 60$ min, the slopes of all $\mathrm{R}_{\mathrm{fil}}(\mathrm{t})$ curves abruptly change, jumping up to $0.055 \Omega / \mathrm{min}$. This slope change corresponds to the silicide $\mathrm{W}_{5} \mathrm{Si}_{3}$ precipitation over all the central part of the filament. Also, even if it is not clear how this transition nucleates, it seems clear that it is rapidly affecting all the catalyzer central part. It was possible to estimate $t_{c} \approx 100$ min by means of Scanning Electron Microscope (SEM) images and Energy-dispersive X-ray spectroscopy (EDX) measurements of the cross section of aged filaments. The details of these measurements are part of a more extended publication over this subject [17]. Using $t_{c} \approx 100 \mathrm{~min}, L_{f i l}=30 \mathrm{~cm}$, and the estimated values for $R_{W}^{\prime}, R_{W S i_{2}}^{\prime}$ and $R_{W_{5} S i_{3}}^{\prime}$ (calculated in section 2) in Eq. 5, it was found a slope in the range of $0.04-0.07 \Omega / \mathrm{min}$ for the signature of $R_{f i l}(t)$ during the $\mathrm{W}_{5} \mathrm{Si}_{3}$ precipitation phase, which includes the one observed experimentally. 
Once the different stages of the chemical change of the filament have been identified by means of the electrical resistance measurements, it is interesting to compare them with the variation of the degradation of the catalytic activity. Fig. 4 shows the normalized thickness $d$ of samples deposited at subsequent intervals of 15 min, for one tested filament, and at intervals of $25 \mathrm{~min}$, for the other two filaments. The first two samples deposited at intervals of $15 \mathrm{~min}$, show exactly the same thickness. This means that at least for the first 30 min the catalytic activity is perfectly preserved.

Hence, a slight decrease of the catalytic activity is observed before any silicides start affecting the central part of the filament. This becomes apparent since it is observed a decrease of less than $10 \%$ in the thickness of the deposited films. However, the most remarkable drop in the catalytic activity is observed just after the beginning of the $\mathrm{W}_{5} \mathrm{Si}_{3}$ precipitation at $t=t_{0} \approx 60 \mathrm{~min}$. This includes a reduction of about $35 \%$ in the thickness of the deposited film, which is partially due to a drop of the filament temperature, caused by the emissivity change. However, by estimating the catalytic activity drop caused by the decrease in temperature from $1900{ }^{\circ} \mathrm{C}$ to $1750{ }^{\circ} \mathrm{C}$, it is found a maximum reduction of only $20 \%$. This result was obtained using $60 \mathrm{~kJ} / \mathrm{moles}$ for the activation energy $E_{a}$ of the silane decomposition [18]. This means that the chemical change from $\mathrm{W}$ to $\mathrm{W}_{5} \mathrm{Si}_{3}$ is also relevant for understanding the observed drop of the catalytic activity. In this case, the decrease is probably induced by a reduction of the superficial active sites present over the heterogeneous catalyst.

Afterwards, $d$ tends to increase for larger $\mathrm{SiH}_{4}$ exposure times. This last effect is attributed to an increase in the filaments catalytic area (due to the increment of the filament radius during the growth of the $\mathrm{W}_{5} \mathrm{Si}_{3}$ outer corona). 
From these results, it seems clear that for a filament working at high temperature like in our case, the catalytic activity is only preserved for a short lapse of time and that it drops considerably at $t=t_{0} \approx 60$ min when the macroscopic $\mathrm{W}_{5} \mathrm{Si}_{3}$ precipitation takes place. Given this, it is interesting to discuss the relationship between $t_{0}$ and other physical variables of the system.

We assume that the $\mathrm{W}_{5} \mathrm{Si}_{3}$ precipitation begins simultaneously over the entire filament surface when the silicon concentration at the surface reaches a limit value called $c_{\text {lim }}$. Silicon atoms are accumulating inside the wire with a flow that, in first approximation, can be considered constant. The reason for this is that the initial $\mathrm{Si}$ concentration is zero whereas the final one, $c_{\text {lim }}$, is much smaller than the equilibrium Si concentration calculated from Raoults' law in the case of an ideal solid solution and standard deposition pressures. This constant flow $J_{S i}^{\text {in }}$ can be expressed using the silane dissociation probability $\gamma_{\mathrm{SiH}_{4}}$, the desorption probability $\gamma_{d e s}$, the silane pressure $p_{\mathrm{SiH}_{4}}$ and the gas temperature $T_{\text {gas }}$ :

$$
J_{S i}^{i n}=\left(1-\gamma_{d e s}\right) \gamma_{S i H_{4}} \frac{p_{S i H_{4}}}{\sqrt{2 \pi m k_{B} T_{g a s}}}
$$

Where, $m$ is the silane molecules mass and $k_{B}$ is the Boltzmann's constant. The accumulated silicon diffuses inside the filament bulk. However, since the diffusion coefficient of silicon in tungsten is extremely low $D \approx 10^{-11}-10^{-12} \mathrm{~cm}^{2} / \mathrm{s}$, about $10^{4}$ times smaller than the diffusion coefficient in the silicided compound [19], Si atoms are accumulating just within a small distance $\lambda$ from the surface of the filament 


$$
\lambda=\frac{\pi D c_{\text {lim }}}{J_{S i}^{i n}}
$$

By solving the diffusion problem [20] for the fulfilled limits $r_{0}^{2} \gg D t$ and $r_{0} \gg \lambda$, it is found that the beginning of the macroscopic $\mathrm{W}_{5} \mathrm{Si}_{3}$ precipitation

$$
t_{0}=\frac{2 m \pi^{2} c_{\text {lim }}^{2} D k_{B} T_{g a s}}{\left(\left(1-\gamma_{\text {des }}\right) \gamma_{\text {SiH }_{4}} p_{\text {SiH }_{4}}\right)^{2}}
$$

does not depend on the radius of the filament in most practical situations because $\lambda \approx 10^{-3}-10^{-4} \mathrm{~cm}$, for $t_{0} \approx 10^{3} \mathrm{~s}, D \approx 10^{-11}-10^{-12} \mathrm{~cm}^{2} / \mathrm{s}$ and $c_{\text {lim }} \approx 10^{21} \mathrm{at} / \mathrm{cm}^{3}$.

Unfortunately, it seems that $t_{0}$ strongly depends on the partial pressure of silane. However, this is a first result which does not take into account the effect of hydrogen on the catalyst aging, which, as we show in a more extended publication [17], becomes relevant for deposition conditions compatible with $\mu \mathrm{c}-\mathrm{Si}: \mathrm{H}$ deposition.

\section{Conclusion}

In this paper, the ageing process of thin tungsten filaments of $0.125 \mathrm{~mm}$ diameter, heated at a high temperature of $1900{ }^{\circ} \mathrm{C}$, under silane partial pressures of $2 \mathrm{~Pa}$ compatible with a-Si:H deposition was studied. In particular, the study was focused on enlightening the relationship between the deterioration of the catalytic activity and the change of the chemical composition of the filament surface. The stability of the catalyst was simply checked by comparing the thickness of films deposited during regular subsequent intervals. To deduce the chemical composition of the filament surface during these deposition intervals, it was developed a model that allowed interpreting the 
filaments electric resistance variation observed during their aging process. The aging tests effectuated over three filaments showed a similar behavior. The catalytic activity remained unchanged for an initial short time comprised between $30 \mathrm{~min}$ and $45 \mathrm{~min}$ during which, silicon is dissolving at the surface of the catalyst. At $t=t_{0} \approx 60 \mathrm{~min}$, the dissolution limits was attained and a macroscopic precipitation of $\mathrm{W}_{5} \mathrm{Si}_{3}$ affected the entire central part of the filament. An abrupt change on the filament catalytic activity was revealed by a decrease of about $35 \%$ in the thickness of the film deposited just after the silicide precipitation. This can be explained by the concurrence of a diminution of the filament temperature caused by the increasing of the emissivity, with the chemical change from $\mathrm{W}$ to $\mathrm{W}_{5} \mathrm{Si}_{3}$ of the catalyst surface. The catalytic lifetime of the filament can be related to $t_{0}$. Due to the extremely small values of the diffusion coefficient of silicon in tungsten, it seems that before any silicide precipitation occurs, the silicon is dissolved only in a superficial layer of few micrometers length from the surface of the catalyst. This is a clear indication that in most practical situations the catalytic stability of a filament does not depend on its radius.

\section{Acknowledgements}

This research has been supported by the Spanish Ministerio de Ciencia e Innovación and the European Regional Development Fund through the projects AMIC (ENE2010-21384-C4-03) and INNDISOL (IPT-420000-2010-006).

\section{References}

1. C.H.M. van der Werf, H. Li, V. Verlaan, C.J. Oliphant, R. Bakker, Z.S. Houweling, R.E.I. Schropp, Thin Solid Films 517(2009)3431. 
2. N. Kniffler, A. Pflueger, D. Scheller, B. Schroeder, Thin Solid Films 517 (2009) 3424.

3. D. Hrunsky, M. Scheib, M. Mertz, B. Schroeder, Thin Solid Films 517(2009) 3370 .

4. D. Knoesen, C. Arendse, S. Halindintwali, T. Muller, Thin Solid Films $516(2008) 822$

5. P.A.T.T. van Veenendaal, O.L.J. Gijzeman, J.K. Rath, R.E.I. Schropp, Thin Solid Films 395(2001) 194.

6. J.K. Holt, M. Swiatek, G. Goddwin, H.A. Atwater, Journal of AppliedPhysics 92(2002) 4803.

7. E. Iwanickzko, A.H. Mahan, B. Yan, L.N. Gedvilas, D.L. Williamson, B.P. Nelson, Materials Research Society Symposium Proceedings 762(2003) A6.9.

8. K. Honda, K. Ohdaira, H. Matsumura, Japanese Journal of Applied Physics 47 (2008) 3692.

9. C. H. M. van der Werf, P.A.T.T van Veenendaal, M.K. van Veen, A.J. Hardeman, M.Y.S. Rusche, J.K. Rath, R.E.I. Schropp, Thin Solid Films $430(2003) 46$.

10. H. Matsumura, Thin Solid Films 395(2001) 1.

11. K. Ishibashi, Thin Solid Films 395(2001) 55.

12. P. A. Frigeri, O. Nos, S. Bengoechea, C. Frevert, J. M. Asensi, J. Bertomeu, Thin Solid Films 517(2009) 3427.

13. P. D. Desai, T. K. Chu, H. M. James, and C. Y. Ho, J. Phys. Chem. Ref. Data, 13 (1984) 1069. 
14. E. Lassner, W. D. Schubert, Tungsten, Kluwer Academic. Plenum Publishers, New York, 1999.

15. F. Nava, K.N. Tu, E. Mazzega, M. Michelini, G. Queirolo, Journal of Applied Physics 61(1987) 1085.

16. C. Gelain, A. Cassuto, P.L. Go, Oxidation of Metals 3 (1971) 115.

17. O. Nos, P. A. Frigeri, J. Bertomeu, Mater. Chem. Phys. To be published.

18. H. L Duan, S.F Bent, Thin Solid Films 485 (2005) 126.

19. H. Jehn, G. Bar, E. Best, E. Koch, B. R. Gudrun, Gmelin Handbook of inorganic Chemistry, vol. A 5b, Springer Berlin, 1993.

20. J. Crank, The Mathematics of Diffusion, Clarendon Press, Oxford, 1979.

\section{Figure Captions}

Fig. 1: Three pictures taken during the degradation test of a $0.125 \mathrm{~mm}$ diameter tungsten filament, heated at $1900{ }^{\circ} \mathrm{C}$, in a pure silane atmosphere at a pressure of $2 \mathrm{~Pa}$. A $\mathrm{WSi}_{2}$ filament portion expanding from the cold end can be identified by looking at the progressive advance of the portion of filament with lower brightness.

Fig. 2: Schematic view of the different regions considered in the proposed model of the filaments electric resistance at different time: $a . \mathrm{WSi}_{2}$ filament portion expanding from the cold end towards the middle of the filament; $b$. transition zone composed of the $\mathrm{WSi}_{2}$ front; c. central part of the filament wherein pure $\mathrm{W}$ is progressively transformed into $\mathrm{W}_{5} \mathrm{Si}_{3}$ for $t>t_{0}$. 
Fig. 3: The electrical resistance, $R_{f i l}$, as a function of time of three different tungsten filaments of $0.125 \mathrm{~mm}$ heated at $1900{ }^{\circ} \mathrm{C}$ aged in a pure silane atmosphere at a pressure of $2 \mathrm{~Pa}$. It has been used the same line style than in Fig. 4 to identify the data taken during the same test.

Fig. 4: Normalized thickness, $d$, of the films deposited during the ageing tests of three filaments. For each filament, different films have been deposited during regular subsequent intervals (15 min for the thick continuous line and $25 \mathrm{~min}$ for the dotted lines). It has been used the same line style than in Fig. 3 to identify the data taken during the same test. 
Fig. 1
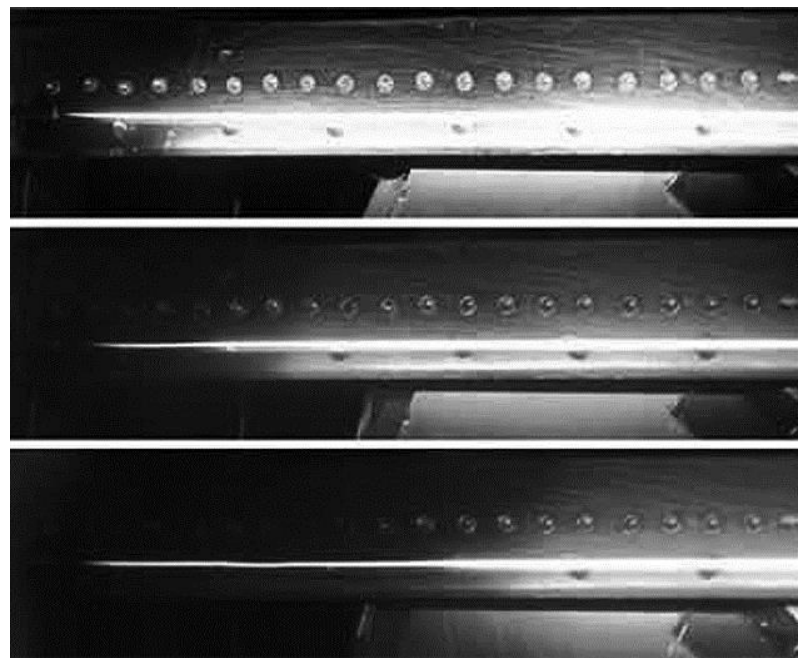

Fig. 2

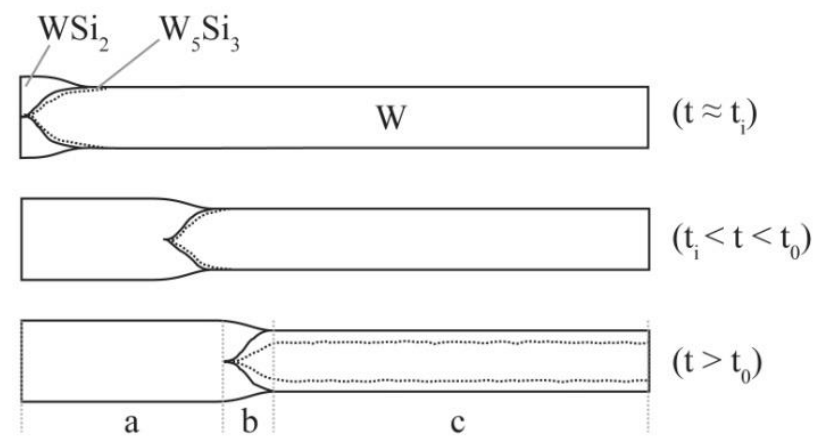


Fig. 3

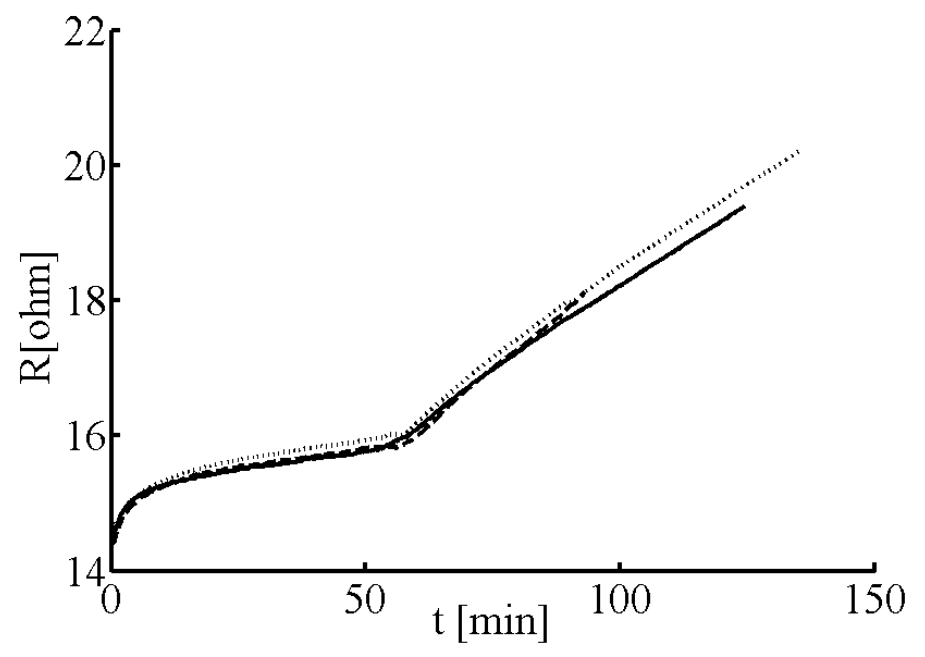

Fig. 4

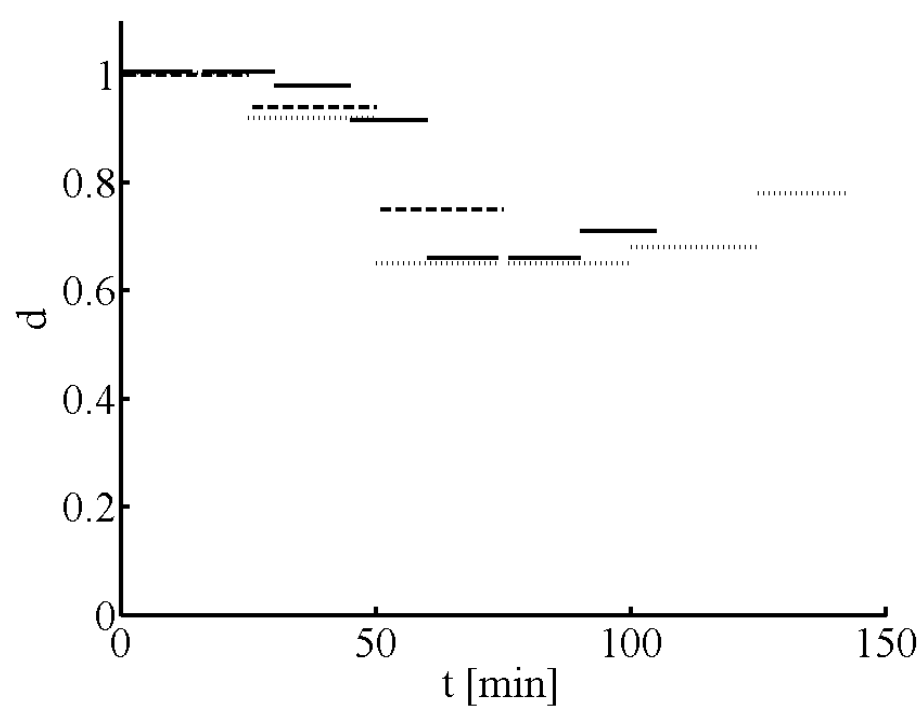

